\title{
SOME ESTIMATIONS IN THE TOPOLOGY OF SIMPLY-CONNECTED ALGEBRAIC SURFACES
}

\author{
BY B. MOISHEZON \\ Communicated by R. T. Seeley, November 10,1976
}

The simplest nontrivial oriented topological surface is the 2-dimensional torus. It is well known that any compact Riemann surface is topologically equivalent to the 2-sphere with handles attached, that is, to a connected sum of 2-toruses. We can consider this decomposition as corresponding to the canonical decomposition of the (skew-symmetric) intersection form of 1-homologies on the given Riemann surface.

In the case of simply-connected algebraic surfaces the intersection form of 2-homologies plays a fundamental role because it defines completely the homotopy type of the corresponding 4-dimensional topological manifold (see [1], [2]).

Performing a $\sigma$-process on the given simply-connected algebraic surface $V$ we obtain an algebraic surface $V^{\prime}$ which contains a 2-dimensional homology class with self-intersection equal -1 (which is an odd number). Then it is well known (see [3], [4]) that there exists a basis of $\mathrm{H}_{2}\left(V^{\prime}, \mathrm{Z}\right)$ such that the corresponding intersection matrix is diagonal. The corresponding "elementary blocks" $\|+1\|$ and $\|-1\|$ are the intersection matrices of the simplest nontrivial oriented simplyconnected 4-manifolds:

$P=$ complex projective plane with its usual orientation and $Q=$ complex projective plane with orientation opposite to the usual. From the homotopy classification theorem [1], [2], it follows that $V^{\prime}$ is homotopy equivalent to a connected sum of $P$ 's and $Q$ 's. Of course, the "ideal situation" (analogous to the mentioned above topological decomposition of compact Riemann surfaces), which we could expect, is the existence of a homeomorphism of $V^{\prime}$ to this connected sum. However, there are some nondirect indications that $V^{\prime}$ is homeomorphic to a connected sum of $P$ 's and $Q$ 's if and only if $V^{\prime}$ is a rational algebraic surface. (This conjecture was formulated in [5].) The question is still open, but assuming the conjecture we can consider as a realistic aim only the problem of estimating how "far" topologically is the given nonrational simply-connected algebraic surface from an "ideal" topological model, that is, from a connected sum of $P$ 's and $Q$ 's.

In [6] Wall proved the following theorem: If $M_{1}, M_{2}$ are simply-connected compact 4-manifolds, which are homotopically equivalent, then there exists

AMS (MOS) subject classifications (1970). Primary 57A15, 14J99, 32J15. 
an integer $k \geqslant 0$ such that $M_{1} \# k\left(S^{2} \times S^{2}\right)$ is diffeomorphic to $M_{2} \#$ $k\left(S^{2} \times S^{2}\right)$ (\# is is the connected sum operation).

It follows almost immediately from this result that if $M$ is a simply-connected compact 4-manifold, then there exists an integer $k \geqslant 0$ such that $M \#$ $(k+1) P \# k \cdot Q$ is diffeomorphic to $l P \# m Q$ for some $l, m \geqslant 0$.

After the proof of his theorem Wall writes the following [6, p. 147]: "We remark that our results is a pure existence theorem; We have obtained, even in principle, no bound whatever on the integer $k$ ".

As it was remarked in [5], the operation $M \# P$ (resp. $M \# Q$ ) where $M$ is an oriented 4-manifold could be considered as performing of certain blowing up of some point on $M$. We call this blowing up $\bar{\sigma}$-process (resp. $\sigma$-process). (The exact definition of $\sigma$-process and $\bar{\sigma}$-process is the following: In a small enough neighborhood $N_{x}$ of a point $x \in M$ we can always take local coordinates giving $N_{x}$ a complex structure. This complex structure will then have an orientation the same as that of $M$ or opposite to that of $M$. Performing a classical $\sigma$-process using the local complex coordinates of $N_{x}$, we get an operation which in the first case we call " $\sigma$-process" and in the second case " $\bar{\sigma}$-process".)

We say that an oriented simply-connected 4-manifold $W$ is completely decomposable (resp. almost completely decomposable) if $W$ (resp. $W \# P$ ) is diffeomorphic to $l P \# m Q$ for some $l, m \geqslant 0$.

Let $M$ be an oriented compact simply-connected 4-manifold. For $\left(k_{1}, k_{2}\right)$ $\in \mathbf{Z} \times \mathbf{Z}, k_{1} \geqslant 0, k_{2} \geqslant 0$, let $M\left(k_{1}, k_{2}\right)$ be a 4-manifold obtained from $M$ by $k_{1} \bar{\sigma}$-processes and $k_{2} \sigma$-processes. Denote by $\mathcal{W}(M)=\left\{\left(k_{1}, k_{2}\right) \in \mathbf{Z} \times \mathbf{Z} \mid k_{1} \geqslant\right.$ $0, k_{2} \geqslant 0, M\left(k_{1}, k_{2}\right)$ is completely decomposable $\}$. It follows from the theorem of Wall that $\omega(M) \neq \varnothing$.

An important geometrical problem is to define minimal elements of $W(M)$ (in any natural sense). A certain step for solving this problem could be the construction of some elements of $W(M)$ in explicit form, say in terms of the 2-dimensional Betti number and of the signature of $M$. We can prove that such a construction is possiule when $M$ admits a complex structure. The main result is

THEOREM A. Let $M$ be a compact simply-connected 4-manifold which admits a complex structure. Take an orientation on $M$ corresponding to certain complex structure on it. Let $K(X), L(X)$ be cubic polynomial defined as follows:

$$
K(X)=\widetilde{K}(9(5 X+4))-X, \quad L(X)=\tilde{L}(9(5 X+4)),
$$

where $\widetilde{K}(t)=t\left(t^{2}-6 t+11\right) / 3, \widetilde{L}(t)=(t-1)\left(2 t^{2}-4 t+3\right) / 3$

$$
\begin{aligned}
& \left(K(X)=30375 X^{3}+68850 X^{2}+52004 X+13092\right. \\
& \left.L(X)=60750 X^{3}+141750 X^{2}+110265 X+28595\right) .
\end{aligned}
$$

Denote by $b_{+}\left(\right.$resp. $\left.b_{-}\right)$the number of positive (resp. negative) squares in the inter- 
section form of $M$ and let $k_{1}^{\prime}=K\left(b_{+}\right), k_{2}^{\prime}=\max \left(0, L\left(b_{+}\right)-b_{-}\right)$. Then the pair $\left(k_{1}^{\prime}, k_{2}^{\prime}\right) \in \omega(M)$.

Remarks about the proof of Theorem A. From the Kodaira classification of compact complex surfaces [7] it follows that if $M$ is a simply-connected compact complex surface, then there exists a nonsingular projective-algebraic complex surface $V$ such that $V$ is diffeomorphic to $M$ and one of the following three possibilities holds:

(a) $V$ is rational;

(b) $V$ is elliptic;

(c) $V$ is of general type.

In case (a) our theorem is evident. In case (b) we can prove a much stronger result.

THEOREM B. Any simply-connected elliptic surface $V$ is almost completely decomposable. (That is, $(1,0) \in W(V)$.)

For the case (c) we first prove the following comparison theorem for topology of projective algebraic surface of given degree $n$ and nonsingular hypersurface of degree $\mathrm{n}$ in $\mathbf{C} P^{3}$ :

THEOREM C. Let $V_{n}$ be a projective algebraic surface of degree $n$ embedded in $\mathbf{C} P^{N}, N \geqslant 5$, such that $V_{n}$ is not contained in a proper projective subspace of $\mathbf{C} P^{N}$. Suppose that $V_{n}$ is nonsingular or has as singularities only rational doublepoints. Let $h: \widetilde{V}_{n} \rightarrow V_{n}$ be a minimal desingularization of $V_{n}$ (that is, $\widetilde{V}_{n}$ has no exceptional curve of first kind s such that $h(s)$ is a point on $V_{n}$ ). Suppose $\pi_{1}\left(\widetilde{V}_{n}\right)=0$. Denote by $Y_{n}$ the diffeomorphic type of a nonsingular hypersurface of degree $n$ in $\mathbf{C} P^{3}$.

Then

(i) $b_{+}\left(\widetilde{V}_{n}\right)<b_{+}\left(Y_{n}\right), b_{-}\left(\widetilde{V}_{n}\right)<b_{-}\left(Y_{n}\right)$;

(ii) $\widetilde{V}_{n} \#\left[b_{+}\left(Y_{n}\right)-b_{+}\left(\widetilde{V}_{n}\right)+1\right] P \#\left[b_{-}\left(Y_{n}\right)-b_{-}\left(\widetilde{V}_{n}\right)\right] Q$ is diffeomorphic to $Y_{n} \# P$.

In [5] it was proved that $Y_{n} \# P$ is completely decomposable. Thus we need only some estimation of a possible minimal degree for projective embeddings of $V$ in terms of $b_{+}(V), b_{-}(V)$. We obtain such an estimation from Bombieri's results on pluricanonical embeddings of algebraic surfaces of general type [8].

ReMARK To Theorem B. Note that Theorem B together with results of [5], [9], [10] show that all big explicit classes of simply-connected algebraic surfaces considered until now have the property that their elements are almost completely decomposable 4-manifolds. That is, the "theoretical" Thereom A gives much weaker results than our "empirical" knowledge.

The interesting question is, how far we can move with such "empirical achievements" in more general classes of simply-connected algebraic surfaces. 


\section{BIBLIOGRAPHY}

1. L. S. Pontryagin, On the classification of four-dimensional manifolds, Uspehi Mat. Nauk. (N.S.) 4 (1949), no. 4 (32), 157-158. (Russian) MR 11, 194.

2. J. H. C. Whitehead, On simply connected, 4-dimensional polyhedra, Comment. Math. Helv. 22 (1949), 48-92. MR 10, 559.

3. J. W. Milnor and D. Husemoller, Symmetric bilinear forms, Ergebnisse Math Grenzgebiete, Band 73, Springer-Verlag, Berlin and New York, 1973.

4. J.-P. Serre, Forms bilinéaires symétriques entières à discriminant \pm 1 , Séminaire Henri Cartan, 1961/62, Exposé 14, Secrétariat mathématique, Paris, 1964. MR 28 \#3443.

5. R. Mandelbaum and $\mathrm{B}$. Moishezon, On the topological structure of non-singular algebraic surfaces in $C P^{3}$, Topology $15 \overline{(1976) .}$

6. C. T. C. Wall, On simply-connected 4-manifolds, J. London Math. Soc. 39 (1964), 141-149. MR 29 \#627.

7. K. Kodaira, On the structure of complex analytic surfaces, IV, Amer. J. Math. 90 (1968), 1048-1066. MR 39 \#473.

8. E. Bombieri, Canonical models of surfaces of general type, Inst. Hautes Études Sci. Publ. Math. 42 (1973), 171-219. MR 47 \#6710.

9. R. Mandelbaum and B. Moishezon, On the topological structure of simply-connected algebraic surfaces, Bull. Amer. Math. Soc. 82 (1976), 731-733.

10. R. Mandelbaum, Algebraic surfaces and irrational connected sums of four manifolds, Topology (to appear).

DEPARTMENT OF MATHEMATICS, TEL-AVIV UNIVERSITY, RAMAT-AVIV, ISRAEL 